\title{
Optical Response of Ag-Au Bimetallic Nanoparticles to Electron Storage in Aqueous Medium
}

Article in Journal of Nanoscience and Nanotechnology · July 2008

DOI: $10.1166 /$ jnn.2008.157 Source: PubMed

CITATIONS

10

6 authors, including:

\section{Hikmet Sezen}

Helmholtz-Zentrum Berlin

55 PUBLICATIONS 303 CITATIONS

SEE PROFILE

\section{Miguel A Correa-Duarte}

University of Vigo

110 PUBLICATIONS 4,851 CITATIONS

SEE PROFILE

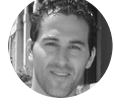

READS

124

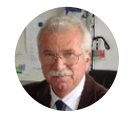

Sefik Suzer

Bilkent University

176 PUBLICATIONS 2,497 CITATIONS

SEE PROFILE

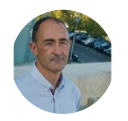

Luis M Liz-Marzán

CIC biomaGUNE

557 PUBLICATIONS $\quad 34,429$ CITATIONS

SEE PROFILE

Some of the authors of this publication are also working on these related projects: 


\title{
Optical Response of Ag-Au Bimetallic Nanoparticles to Electron Storage in Aqueous Medium
}

\author{
Ilknur Tunc ${ }^{1}$, Haci Osman Guvenc ${ }^{1}$, Hikmet Sezen ${ }^{1}$, Sefik Suzer ${ }^{1, *}$, \\ Miguel A. Correa-Duarte², and Luis M. Liz-Marzán² \\ ${ }^{1}$ Chemistry Department, Bilkent University, 06800 Ankara, Turkey \\ ${ }^{2}$ Departamento de Química Física, and Unidad Asociada CSIC-Universidade de Vigo, 36310 Vigo, Spain
}

\begin{abstract}
Composition and structure dependence of the shift in the position of the surface plasmon resonance band upon introduction of $\mathrm{NaBH}_{4}$ to aqueous solutions of gold and silver nanoparticles are presented. Silver and gold nanoalloys in different compositions were prepared by co-reduction of the corresponding salt mixtures using sodium citrate as the reducing agent. After addition of $\mathrm{NaBH}_{4}$ to the resultant nanoalloys, the maximum of their surface plasmon resonance band, ranging between that of pure silver (ca. $400 \mathrm{~nm}$ ) and of pure gold (ca. $530 \mathrm{~nm}$ ), is blue-shifted as a result of electron storage on the particles. The extent of this blue shift increases non-linearly with the mole fraction of silver in the nanoparticle, parallel to the trends reported previously for both the frequency and the extinction coefficient of the plasmon band shifts. Gold(core)@silver(shell) nanoparticles were prepared by sequential reduction of gold and silver, where addition of $\mathrm{NaBH}_{4}$ results in relatively large spectral shift in the plasmon resonance band when compared with the nanoalloys having a similar overall composition. The origin of the large plasmon band shift in the core-shell is related with a higher silver surface concentration on these particles. Hence, the chemical nature of the nanoparticle emerges as the dominating factor contributing to the extent of the spectral shift as a result of electron storage in bimetallic systems.
\end{abstract}

Keywords: Gold and Silver Nanoparticles, Spectral Shifts, Charge Storage, Core-Shell Structure.

\section{INTRODUCTION}

Nanoparticles have drawn an intense attention of chemists, physicists, biologists and engineers due to their unique electronic, optical and chemical properties. ${ }^{1-8}$ In metallic nanoparticles, due to the increased surface-to-volume ratio, new optical properties arise, which are dominated by strong extinction bands, attributed to surface plasmon resonances, and which are in the UV-visible region for some noble metals. Among these, gold and silver nanoparticles are usually the metals of choice because of their very well defined absorption bands and high chemical stability. ${ }^{9-15}$ A major effort has been devoted for investigation of photochemical properties of these materials by following spectral changes in these bands. These spectral changes arise mainly as shifts due to changes in: (i) size, (ii) shape, (iii) composition, and/or (iv) environment.

In particular, size dependence of the plasmon resonance band of gold nanoparticles has been heavily investigated. ${ }^{16-29}$ Silver and gold nanoparticles smaller

*Author to whom correspondence should be addressed. than $3 \mathrm{~nm}$ in diameter do not exhibit significant absorption. However, particles of 3-50 nm show a very strong absorption band at 390-420 and 520-560 nm for silver and gold respectively. ${ }^{21,22}$ As the particles' sizes increase, absorption bands broaden and shift to higher wavelengths (redshift), ${ }^{16,17,25}$ and higher order modes can be excited. ${ }^{29}$ The well-defined plasmon absorption bands of silver and gold show particle-shape-dependence as well. For example, as the shape of silver nanoparticles changes from spherical to triangular prism, various modes can be identified, with the in-plane dipole band strongly red-shifted as compared to that of small spheres $(400 \mathrm{~nm}){ }^{30} \mathrm{Gold} /$ silver core-shell nanoparticles exhibit drastic changes in the location of their surface plasmon resonance bands, strongly depending on whether gold or silver is placed as shell, such that, the shell mostly contributes to the energy of the plasmon resonance band. ${ }^{31}$

Alloying is another synthetic tool that can be used to induce shifts of the plasmon band, as was first shown by Link et al. for bimetallic nanoparticles containing gold and silver, where the absorption band was shown to be tunable between $380-530 \mathrm{~nm}$, by varying only the nanoparticle 
composition. ${ }^{32}$ The presence of a single absorption band for each sample and the observed changes in extinction coefficient, both of which shifted linearly with increasing mole fraction of the gold were taken as the evidence for alloy formation as opposed to core-shell type of structure. These findings were later substantiated by additional structural and other spectroscopic investigations. ${ }^{33,34}$

Addition of reducing agents such as borohydride ions has been shown to lead to electron injection in metal nanoparticles as was also evidenced by the resulting blue shift. ${ }^{26}$ Presence of electron acceptor agents such as nitrobenzene, methyl viologen, thionine prevents electron storage on metal nanoparticles by preferential capturing of the electrons by themselves. ${ }^{35-37}$ The corresponding positive-charge-storage was also reported by Mulvaney et al. by the observation of the red shift in the plasmon absorption band of colloidal silver particles when nucleophilic reagents (like $\mathrm{I}^{-}$) were present in the solution. ${ }^{38}$

Numerous publications have appeared for predicting these shifts in the absorption spectrum of metallic nanoparticles by Drude Model $^{26,39}$ using the factors which influence the plasmon absorption. The theory emphasizes on two factors; (i) dielectric constant of the medium, and (ii) density of the free electrons on the particle surface, such that storage of additional electrons on the nanoparticles is expected to cause a blue-shift in the spectrum. In a recent study, Kamat et al. showed that silver nanoparticles retain their electron storage capacity even when they are prepared as the core surrounded by titania shell by following the extent of the blue shift when irradiated by UV light. $^{40-42}$

In all previous publications possibility of chargestorage on monometallic nanoparticles, especially on gold and silver, has been discussed. The major idea behind charged particles is the possibility to enhance catalytic/photocatalytic properties of these particles and/or tune the luminescent or sensing properties. Similarly optical properties of the gold-silver alloys and core-shells have also been extensively investigated. However, chargestorage in the silver-gold alloy nanoparticles and/or coreshells has not been reported to date. In this contribution, charging properties of silver-gold alloy and core-shell particles in aqueous media, induced by borohydride, are followed by the shifts of the respective absorption bands.

\section{EXPERIMENTAL DETAILS}

$\mathrm{HAuCl}_{4}, \mathrm{AgNO}_{3}$, trisodium citrate dihydrate, $\mathrm{NaBH}_{4}$, L-Ascorbic Acid, and CTAB (cetyltrimethylammonium bromide) were purchased from Aldrich and used without further treatment. Milli-Q grade water was used in all preparations. Charging (optical) properties of alloy nanoparticles were characterized by a Cary $5 \mathrm{E}$ UV-Vis-NIR spectrophotometer. To study the charge injection to the nanoparticles, freshly prepared ice-cool aqueous borohydride solution was added, and changes in the spectra were recorded. Transmission Electron Microscope (TEM) images were obtained with a JEOL JEM-1010 microscope operating at $100 \mathrm{kV}$.

The method for preparation of silver-gold nanoalloys was based on co-reduction of $\mathrm{HAuCl}_{4}$, and $\mathrm{AgNO}_{3}$, as reported elsewhere. ${ }^{34}$ We kept the total metal concentration at $0.5 \mathrm{mM}$, while preparing citrate capped $\mathrm{Ag}-\mathrm{Au}$ alloys in different molar ratios $(15,25,60,80 \% \mathrm{Au})$. To a boiling $\mathrm{HAuCl}_{4}$ solution, $\mathrm{AgNO}_{3}$ solution at the corresponding concentration was added under vigorous stirring. Then, $5 \mathrm{~mL}$ pre-heated citrate $(1 \mathrm{wt} \%)$ solution was added to the boiling mixed solution under vigorous stirring. After 30 min of boiling to ensure completion of the reduction, citrate capped $\mathrm{Ag}-\mathrm{Au}$ alloy nanoparticles were ready to use. Pure silver nanoparticles were prepared according to the previously published procedure, ${ }^{35}$ with some modifications. Briefly, $1 \mathrm{~mL}$ of $0.01 \mathrm{M} \mathrm{AgNO}_{3}$ was rapidly added to $99 \mathrm{~mL}$ of solution containing $1 \mathrm{mM} \mathrm{NaBH}_{4}$ and $0.30 \mathrm{mM}$ sodium-citrate under vigorous stirring at room temperature. The solution turned yellow in few minutes. The same procedure was used for synthesis of pure gold nanoparticles by $\mathrm{NaBH}_{4}$ reduction of $\mathrm{HAuCl}_{4}$ (deep red colloid).

The synthesis of gold(core)@silver(shell) nanoparticles was based on the sequential reduction of gold and silver as reported by Rodríguez-González et al. ${ }^{43}$ In brief, $0.5 \mathrm{~mL}$ of preformed $5 \times 10^{-4} \mathrm{M}$ spherical gold nanoparticles was added to $20 \mathrm{~mL}$ of $50 \mathrm{mM}$ aqueous CTAB solution. Then, $1 \mathrm{~mL}$ of ascorbic acid, $0.5 \mathrm{~mL}$ of $10 \mathrm{mM} \mathrm{AgNO}_{3}$, and $0.1 \mathrm{~mL}$ of $1.0 \mathrm{M} \mathrm{NaOH}$ were added subsequently. Fast color change from red to yellow was taken as the evidence of coverage of silver around gold nanoparticles. The expected silver shell thickness was around $15 \mathrm{~nm}$, as determined by TEM. By manipulating the amount of silver and gold it is possible to obtain core-shell nanoparticles with different compositions.

\section{RESULTS}

Gold and silver nanoparticles form nanoalloys when reduced simultaneously in the same mixture, which can be confirmed by optical spectroscopy. If pure gold and silver nanoparticles were mixed in the same solution, two plasmon bands should be observed, while for alloys a single plasmon band is expected. ${ }^{32,33}$ In Figure 1(a), we display the recorded plasmon resonance absorption bands of different Ag-Au alloys with varying composition. The sizes of the nanoparticles are in the range of $25-35 \mathrm{~nm}$ as determined from their TEM images (not shown). The plasmon bands span the spectral range between $19000 \mathrm{~cm}^{-1}$ $(526 \mathrm{~nm})$ and $25000 \mathrm{~cm}^{-1}(400 \mathrm{~nm})$ corresponding to the pure $\mathrm{Au}$ and pure $\mathrm{Ag}$ nanoparticles, respectively.

By using Mie theory, numerous workers have shown that the plasmon band position as well as its extinction coefficient are both related to the electron density $(n)$, 


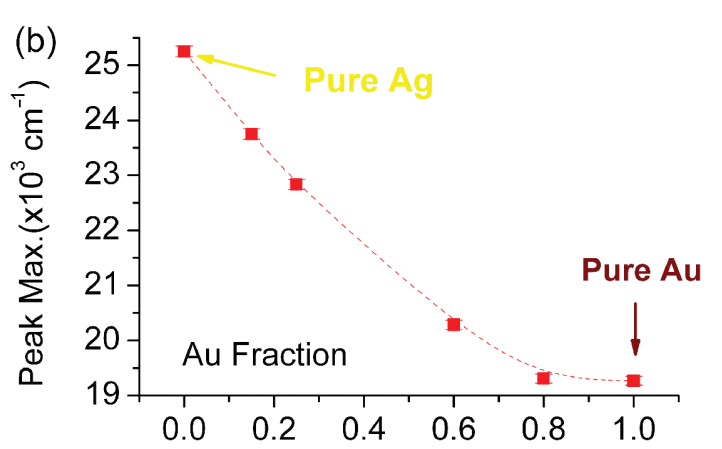

(a)

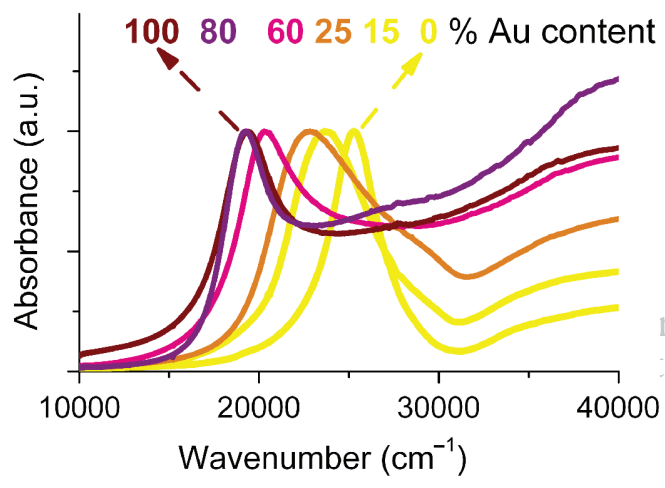

Fig. 1. (a) UV-Vis absorption spectra (plotted in wavenumbers) of pure $\mathrm{Ag}$, pure $\mathrm{Au}$ and their alloys, (b) plot of the peak maximum with respect to Au composition.

the electron effective mass $\left(m_{\text {eff }}\right)$, and the dielectric function $\left(\varepsilon_{2}\right)$ of the medium through the relation: ${ }^{31}$

$$
\omega_{\max }=\frac{n e^{2}}{\varepsilon_{0} m_{\text {eff }} \sqrt{1+\varepsilon_{2}}}
$$

When the maximum position of the alloy plasmon bands is plotted in wavelength scale versus gold mole fraction, a quasi-linear trend has been reported in previous studies. ${ }^{32-34}$ However, when plotted in energy scale (wavenumber) a non-linear trend is obtained as shown in Figure 1(b), which, in this scale, should show a linear dependence if electron density were the sole contributor to the observed spectral shifts. We use the wavenumber scale in all of our figures for meaningful and direct visual comparison with the changes in the plasmon frequency, since a $10 \mathrm{~nm}$ shift around $400 \mathrm{~nm}$ and around $500 \mathrm{~nm}$ correspond to different extent of shifts in the frequency scale.

It was shown by Link et al. that although the theoretical effective mass and the electron densities are very similar for gold and silver, both the positions and the extinction coefficients of the two are very different. ${ }^{32}$ Accordingly, the extinction coefficient of $20 \mathrm{~nm}$ pure silver nanoparticles is approximately 5 times larger than that of pure gold nanoparticles. Furthermore, by using experimentally derived optical parameters from thin films of gold-silver alloys, they were also able to calculate the optical spectrum of gold-silver nanoalloys which closely matched the experimental data. ${ }^{32,33}$

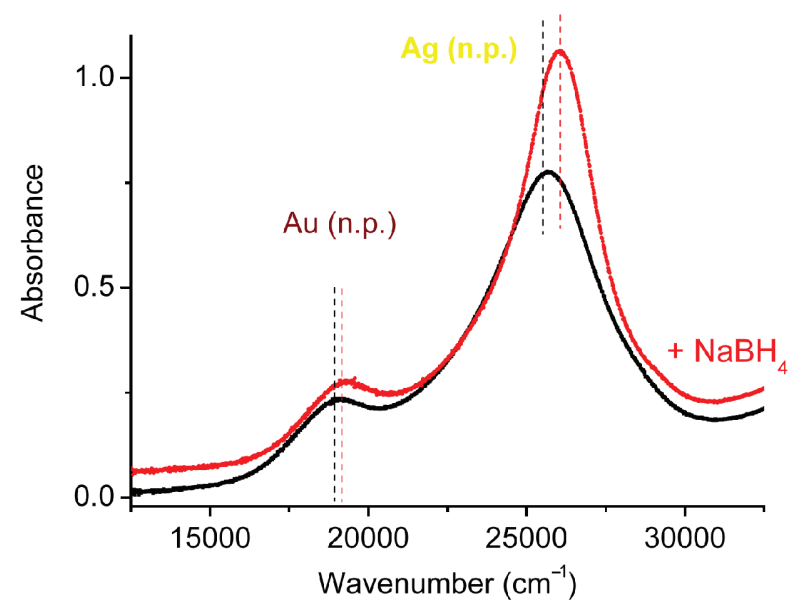

Fig. 2. Spectra of a solution containing pure $\mathrm{Ag}$, and pure $\mathrm{Au}$ nanoparticles having similar size and concentration before and after addition of $\mathrm{NaBH}_{4}$ for electron storage.

In what follows, we will use this finding that, when compared to gold, silver either has a larger electron density or a smaller effective mass to cause both a larger shift in the position of the frequency of the plasmon band and also in its extinction coefficient, to guide us in understanding, qualitatively, the spectral shifts we observe.

\subsection{Effect of Nanoparticle Composition on Electron Injection}

To study charge injection into metal nanoparticles, we have used the oxidation of borohydride, which upon addition to solution, leads to cathodic polarization of the particles, resulting in negatively charged particles. ${ }^{36}$

Although electron addition generally leads to a redshift in optical spectra of molecules, electron injection into metallic nanoparticles causes a blue-shift of the corresponding surface plasmon resonance band, along with an increase in the extinction coefficient. This is best exemplified in Figure 2, which displays the spectra before and after addition of borohydride to a solution containing both silver and gold nanoparticles having similar size and concentration, and was mixed after separate preparation. It is clear from the figure that silver nanoparticles have larger extinction coefficients, as previously reported. ${ }^{33}$ Furthermore, both the blue-shift and the differential change in the extinction coefficient in pure $\mathrm{Ag}$ nanoparticles as a result of electron injection are also larger (shift is ca. $600 \mathrm{~cm}^{-1}$ ) than that of pure $\mathrm{Au}$ (ca. $200 \mathrm{~cm}^{-1}$ ), in close agreement with the predictions from Mie Theory.

Similarly, one can also follow electron storage in the alloy nanoparticles. In Figure 3, we display the spectra before and after addition of exactly the same amount of $\mathrm{NaBH}_{4}$ to the aqueous solutions containing pure Ag, pure $\mathrm{Au}$ and a representative example of alloy nanoparticles (containing 15\% Au). In all cases, the plasmon bands exhibit a blue-shift as a result of additional electrons 


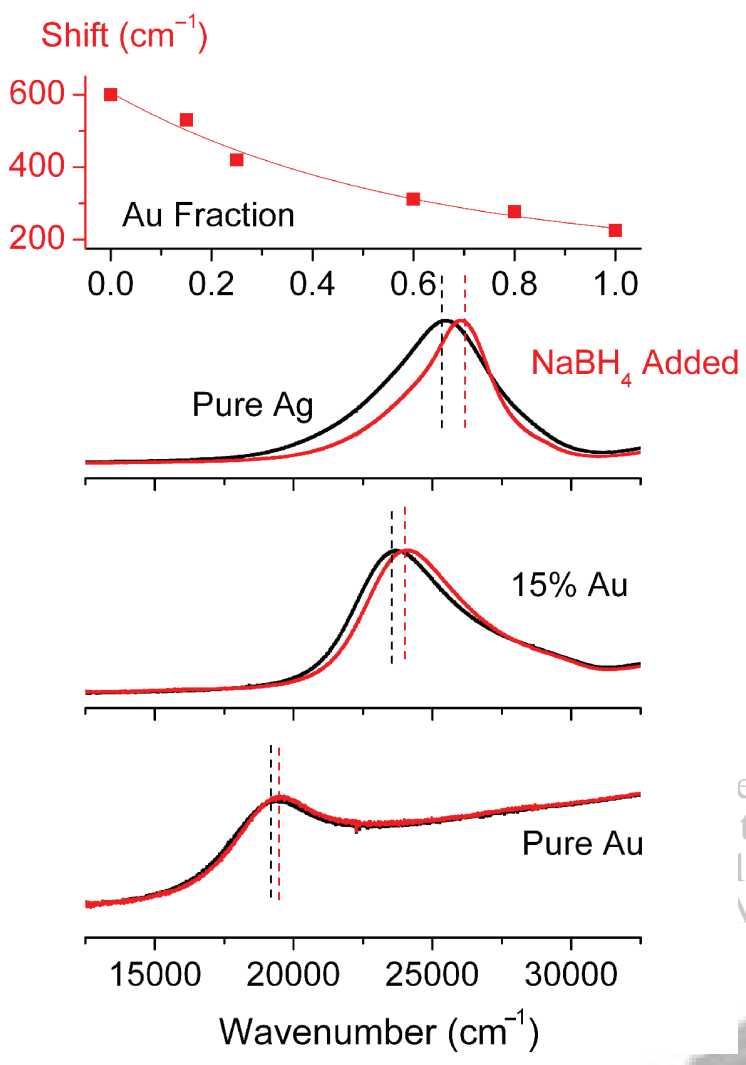

Fig. 3. Spectra of pure Ag, pure $\mathrm{Au}$ and $15 \% \mathrm{Au}$ alloy before and after addition of $\mathrm{NaBH}_{4}$ for electron storage. The top plot gives the shift in the plasmon band with composition upon addition of $\mathrm{NaBH}_{4}$ for electron storage.

stored in the nanoparticles. As shown on the upper plot of Figure 3, the shifts measured for the alloys also exhibit a quasi-linear dependence on $\mathrm{Ag}$ content. Thus, an increase of $\mathrm{Ag}$ content within the alloy, leads to a more extensive blue-shift, in agreement with our practical guideline introduced above.

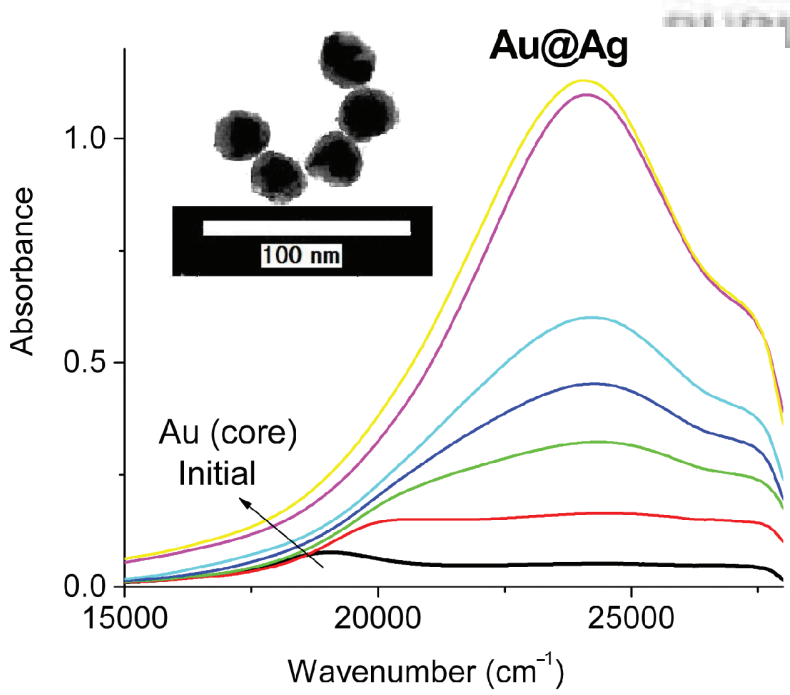

Fig. 4. A sequence of UV-Vis spectra recorded during formation of $\mathrm{Au}$ (core)@ $\mathrm{Ag}$ (shell) nanoparticles. A TEM image of the core-shell particles is given as an inset.

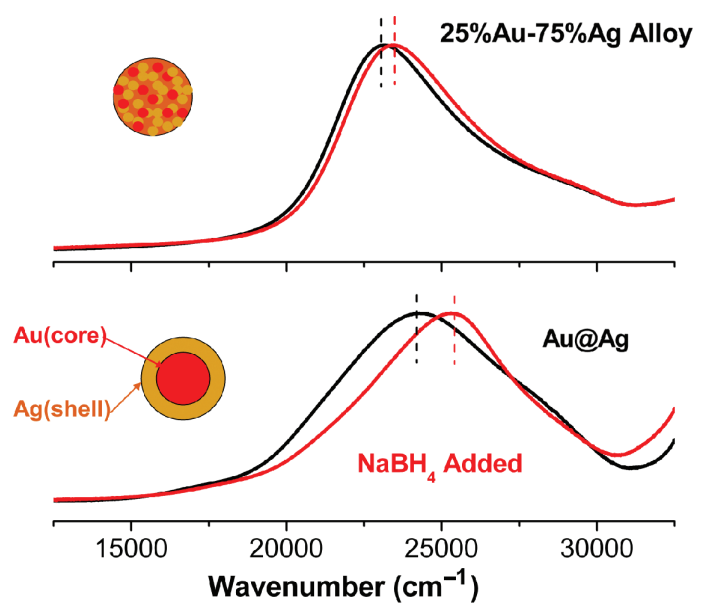

Fig. 5. Spectra of Au-Ag nanoalloys versus $\mathrm{Au}($ core $) @ \mathrm{Ag}($ shell) with $25 \% \mathrm{Au}$ by before and after addition of $\mathrm{NaBH}_{4}$.

\subsection{Effect of Structure on Electron Storage}

The optical response of bimetallic nanoparticles does not only depend on the relative amounts of both metals, but also on the actual distribution within the particles. ${ }^{26} \mathrm{In}$ a similar fashion, it is expected that the effect of charge injection also depends on the structure of Au-Ag nanoparticles. We present here a comparison of the optical effects observed upon charge injection on $\mathrm{Au}-\mathrm{Ag}$ nanoalloys versus core-shell nanoparticles. In Figure 4, a sequential formation of $\mathrm{Au}($ core $) @ \mathrm{Ag}$ (shell) is depicted by reduction of $\mathrm{Ag}$ on $\mathrm{Au}$ (core) which is followed by the plasmon band shift from $530 \mathrm{~nm}$ to $400 \mathrm{~nm}$. Drastic band-shift is an evidence of formation of the silver shell, because it is the shell atoms which mostly contribute to the surface plasmon band. ${ }^{31,43}$

Figure 5 displays the plasmon band shifts for $\mathrm{Au}-\mathrm{Ag}$ nanoalloys, as compared to those for $\mathrm{Au}(\mathrm{core}) @ \mathrm{Ag}($ shell), both with a nominal $25 \%$ Au content. As a result of charge storage upon $\mathrm{NaBH}_{4}$ addition, the plasmon bands of the alloy and the Au@Ag are blue-shifted by $350 \mathrm{~cm}^{-1}$ and $1000 \mathrm{~cm}^{-1}$, respectively. Such a large difference is undoubtedly related with the different $\mathrm{Ag}$ and $\mathrm{Au}$ allocation within these nanoparticles. Although conduction electrons of the $\mathrm{Ag}$ and $\mathrm{Au}$ atoms move freely within the whole cluster in the alloy, those in the core-shell particles are restricted to their part, either nucleus or shell. ${ }^{31}$ In the case of the alloy, both $\mathrm{Au}$ and $\mathrm{Ag}$ contribute to the plasmon absorption frequency. However, in the core-shell case it is the silver shell which contributes most, and in agreement with our assessment earlier in the text, causes a larger spectral shift.

\section{DISCUSSION AND CONCLUSION}

Electron injection into nanoparticles is an important process, since it can affect many properties (chemical, catalytic, optical, magnetic, etc.) for numerous applications. 
Therefore, simple and reliable methods for assessing the extent of the charge injected are required, and spectral shifts in the plasmon position may offer such an opportunity. However, many parameters, like the chemical nature of the particle, its size, type, structure, surrounding medium, etc. contribute to these spectral shifts, and sorting them out one by one is a grand challenge.

By combining electrochemistry with spectroscopy Ung et al. reported that pure $\mathrm{Ag}$ particles of $11 \mathrm{~nm}$ of average size displayed ca. $600 \mathrm{~cm}^{-1}$ blue-shift when they were cathodically polarized, and using the change in the electrode potential they claimed that this corresponded ca. 2000 electrons stored on each nanoparticle. ${ }^{44}$ Using a different approach and by titrating with a stronger electron capturer, thionine, Kamat et al. reported a more than $1000 \mathrm{~cm}^{-1}$ blue-shift in the band position of the $\mathrm{Ag}($ core $) @ \mathrm{TiO}_{2}$ (shell) particles when subjected to photoinduced electron transfer to the silver core of ca. $4 \mathrm{~nm}$ size. They claimed that this spectral shift corresponded to ca. 66 electrons stored in the silver core. ${ }^{35}$.41 42 Another spectroelectrochemical study reports ca. $80 \mathrm{~cm}^{-1}$ spectral shift corresponding to only 1 additional electron of ca. $2 \mathrm{~nm}$ gold particles. ${ }^{45}$

It is worth emphasizing that those shifts reported in these 3 studies as a result of electron storage in the nanoparticles are very close to our experimental findings (ca. $100 \mathrm{~cm}^{-1}$ and $600 \mathrm{~cm}^{-1}$ shifts for $\mathrm{Au}$ and $\mathrm{Ag}$, respectively), but the estimated number of electrons vary significantly from 1 to 2000 electrons. Although we can not estimate the number of electrons stored in the bimetallic particles, our findings point out that the chemical nature of the nanoparticle is the dominating factor contributing to the extent of the spectral shift in bimetallic systems, and silver is approximately 4-5 times more effective when compared to gold.

Acknowledgments: This work was partially supported by TUBA (Turkish Academy of Sciences) and by TUBITAK (The Scientific and Technological Research Council of Turkey) through the grant no. 105M094, and by the Spanish Ministerio de Educación y Ciencia/FEDER through grant no. MAT2004-02991.

\section{References and Notes}

1. D. V. Goia and E. Matijevic, New J. Chem. 22, 1203 (1998).

2. A. Badia, R. B. Lennox, and L. Reven, Acc. Chem. Res. 33, 475 (2000).

3. C. L. Haynes and R. P. Van Duyne, J. Phys. Chem. B 105, 5599 (2001).

4. J. H. Hodak, A. Henglein, and G. V. Hartland, J. Phys. Chem. B 104, 9954 (2000).

5. S. Link and M. A. El-Sayed, J. Phys. Chem. B 103, 8410 (1999).

6. A. C. Templeton, M. P. Wuelfing, and R. W. Murray, Acc. Chem. Res. 33, 27 (2000).
7. R. L. Whetten, M. N. Shafigullin, J. T. Khoury, T. G. Schaaff, I. Vezmar, M. M. Alvarez, and A. Wilkinson, Acc. Chem. Res. 32, 397 (1999)

8. X. M. Zhao, Y. N. Xia, and G. M. Whitesides, J. Mater. Chem. 7, 1069 (1997).

9. J. Y. Bigot, J. C. Merle, O. Cregut, and A. Daunois, Phys. Rev. Lett. 75, 4702 (1995).

10. M. J. Bloemer, J. W. Haus, and P. R. Ashley, J. Opt. Soc. Am. B 7, 790 (1990).

11. R. H. Doremus, J. Chem. Phys. 40, 2389 (1964).

12. E. J. Heilweil and R. M. Hochstrasser, J. Chem. Phys. 82, 4762 (1985).

13. G. C. Papavassiliou, Prog. Solid State Chem. 12, 185 (1979).

14. J. A. A. J. Perenboom, P. Wyder, and F. Meier, Phys. Rep. 78, 173 (1981).

15. T. W. Roberti, B. A. Smith, and J. Z. Zhang, J. Chem. Phys. 102, 3860 (1995).

16. M. M. Alvarez, J. T. Khoury, T. G. Schaaff, M. N. Shafigullin, I. Vezmar, and R. L. Whetten, J. Phys. Chem. B 101, 3706 (1997).

17. S. W. Chen, R. S. Ingram, M. J. Hostetler, J. J. Pietron, R. W. Murray, T. G. Schaaff, J. T. Khoury, M. M. Alvarez, and R. L. Whetten, Science 280, 2098 (1998).

18. M. A. El-Sayed, Acc. Chem. Res. 34, 257 (2001).

19. J. R. Heath, C. M. Knobler, and D. V. Leff, J. Phys. Chem. B 101, 189 (1997).

20. A. Henglein, PCCP 99, 903 (1995).

21. A. Henglein, Langmuir 15, 6738 (1999).

22. A. Henglein and D. Meisel, Langmuir 14, 7392 (1998).

23. S. H. Kim, G. Medeiros-Ribeiro, D. A. A. Ohlberg, R. S. Williams, and J. R. Heath, J. Phys. Chem. B 103, 10341 (1999).

24. S. Link and M. A. El-Sayed, J. Phys. Chem. B 103, 4212 (1999).

25. C. A. Mirkin, R. L. Letsinger, R. C. Mucic, and J. J. Storhoff, Nature 382, 607 (1996).

26. P. Mulvaney, Langmuir 12, 788 (1996)

27. M. P. Pileni, New J. Chem. 22, 693 (1998).

28. T. G. Schaaff, M. N. Shafigullin, J. T. Khoury, I. Vezmar, R. L. Whetten, W. G. Cullen, P. N. First, C. GutierrezWing, J. Ascensio, and M. J. JoseYacaman, J. Phys. Chem. B 101, 7885 (1997).

29. J. Rodriguez-Fernandez, J. Perez-Juste, F. J. G. de Abajo, and L. M. Liz-Marzan, Langmuir 22, 7007 (2006).

30. R. C. Jin, Y. W. Cao, C. A. Mirkin, K. L. Kelly, G. C. Schatz, and J. G. Zheng, Science 294, 1901 (2001).

31. U. Kreibig and M. Vollmer, Optical Properties of Metal Clusters, Springer, Berlin (1995).

32. S. Link, Z. L. Wang, and M. A. El-Sayed, J. Phys. Chem. B 103, 3529 (1999).

33. M. P. Mallin and C. J. Murphy, Nano Lett. 2, 1235 (2002).

34. B. Rodriguez-Gonzalez, A. Sanchez-Iglesias, M. Giersig, and L. M. Liz-Marzan, Faraday Disc. 125, 133 (2004).

35. M. Jakob, H. Levanon, and P. V. Kamat, Nano Lett. 3, 353 (2003).

36. T. Ung, L. M. Liz-Marzan, and P. Mulvaney, J. Phys. Chem. B 103, 6770 (1999).

37. P. V. Kamat, J. Phys. Chem. B 111, 2834 (2007).

38. P. Mulvaney, T. Linnert, and A. Henglein, J. Phys. Chem. 95, 7843 (1991).

39. P. Mulvaney, J. Perez-Juste, M. Giersig, L. M. Liz-Marzan, and C. Pecharroman, Plasmonics 1, 61 (2006).

40. P. V. Kamat, J. Phys. Chem. B 106, 7729 (2002).

41. T. Hirakawa and P. V. Kamat, Langmuir 20, 5645 (2004)

42. T. Hirakawa and P. V. Kamat, J. Am. Chem. Soc. 127, 3928 (2005).

43. B. Rodriguez-Gonzalez, A. Burrows, M. Watanabe, C. J. Kiely, and L. M. L. Marzan, J. Mater. Chem. 15, 1755 (2005).

44. T. Ung, M. Giersig, D. Dunstan, and P. Mulvaney, Langmuir 13, 1773 (1997).

45. G. C. Lica, B. S. Zelakiewicz, M. Constantinescu, and Y. Y. Tong, J. Phys. Chem. B 108, 19896 (2004).

Received: 9 May 2007. Accepted: 25 September 2007. 